\title{
THE ESTIMATION OF PHENACETIN AND OTHER PARA-AMINOPHENOL DERIVATIVES BY HYPOCHLOROUS ACID.
}

\author{
BY A. D. POWELL.
}

(Read at the Meeting, December 4, 1918.)

THe estimation of the substituted phenetidine compounds used in medicine, either alone or in admixture with other substances such as salol and caffeine, has always presented certain difficulties, and various methods have been proposed in recent years for the analysis of mixtures of these substances. Several of these are based on the varying solubilities of the compounds in organic solvents, a more or less complete separation being made, and the separated substances determined gravimetrically. Thus, Seidell (J. Amer. Chem. Soc., 1907, 29, 1088-1091 ; Anauyst, 1907, 32, 360) proposed a method of this type for the estimation of acetanilide, phenacetin, etc., in "headache powders." Emery, Spencer, and Le Febvre (J. Ind. and. Eng. Chem., 
$1915,5,681-684$; ANalyst, 1915, 40, 445), for the estimation of phenacetin and salol, make use of selective hydrolysis, finally reconverting the phenetidine to phenacetin by acetylation, and weighing the phenacetin as such, the salol being estimated by bromine absorption. Another method recently published by Salkover (Amer. J. Pharm., 1916, 88, 484-485 ; ANaLyst, 1917, '42, 16) for the separation of these drugs depends on the solubility of salol in petroleum ether, phenacetin and acetanilide being nearly insoluble in this solvent.

Such methods suffer from the obvious defect that they do not sufficiently identify the substances present in the mixture, and the melting-points of the separated constituents cannot always be relied on, owing to the separation not being perfect.

Methods taking into account the ohemical constitution of these compounds have also been proposed. Taylor and Vanderkleed (Amer. J. Pharm., 1907, 79, 151-156; AnaLYst, 1907, 32, 215), for instance, estimate phenacetin and acetanilide (individually), by steam distillation and titration of the acetic acid produced on hydrolysis. Another method proposed by Emery ( $J$. Ind. and Eng. Chem., 1914, 4, 665-669; ANalyst, 1914, 39, 433) for the separation and estimation of phenacetin and acetanilide depends on the property of phenacetin of combining with iodine to form an insoluble periodide, acetanilide either not reacting with iodine or producing a soluble compound. The amount of iodine precipitated from solution is determined by titration of the excess left in solution, and the phenacetin content calculated from the figures thus obtained. An iodimetric method for the estimation of phenolic compounds has been published by Wilkie (J. Soc. Chem. Ind., 1911, 30, 398 ; Anauyst, $1911,36,294)$; but this does not appear to have been extended to the aminophenols and phenetidins, although it seems probable that such compounds might form definite iodo-derivatives.

As far as my own experience goes, however, no method has been published in which any characteristic reaction of $p$-phenetidine or $p$-aminaphenol has been taken as the basis for the estimation of this group of compounds. The reactions of $p$ aminophenol with oxidising agents were therefore investigated, in order to determine which, if any, could be made the basis of a quantitative estimation. Oxidation by means of potassium dichromate to form quinone was tried, but was found unsatisfactory, as part of the $p$-aminophenol was converted to quinhydrone. The reaction between sodium hypochlorite and an acid solution of $p$-aminophenol was found to be much more promising. These substances react in accordance with the equation

$$
\mathrm{OH} \cdot \mathrm{C}_{6} \mathrm{H}_{4} \cdot \mathrm{NH}_{2} \mathrm{HCl}+4 \mathrm{Cl}=\mathrm{O}: \mathrm{C}_{6} \mathrm{H}_{4}: \mathrm{NCl}+4 \mathrm{HCl} \text {, }
$$

the quinone chlorimine precipitating as golden-yellow flocks from concentrated solutions, but remaining in solution at dilutions below about 1 per cent.

$P$-phenetidine is also converted to quinone chlorimine by the action of hypochlorous acid. The reaction is quantitative, and provides a rapid means for the estimation of these bases and all their derivatives which yield the free base on hydrolysis.

The direct absorption of chlorine does not form a suitable basis for calculating the results, owing to the difficulty of decermining when an excess has been added, and 
it is therefore necessary to determine the amount of quinone chlorimine formed, after addition of excess of hypochlorite and removal of free chlorine from solution. In the absence of free chlorine, the reaction between the quinone compound and hydriodic acid affords a convenient means of determining this. The reaction is the reverse of that given above, four atoms of iodine being liberated by each molecule of quinone chlorimime, and $p$-aminophenol being re-formed.

As no reagent was found which will combine with the excess of free chlorine without also decomposing the chlorimine, and boiling is out of the question owing to the volatility and instability of the latter in hot solutions, the chlorine must be removed by blowing a current of air through the solution. Experiments showed that chlorine is fairly rapidly removed by this means, 100 c.c. of a saturated aqueous solution of this gas losing 98 per cent. of its strength after five minutes aeration at the rate of 700 to 800 c.c. of air per minute, and becoming practically free from chlorine after fifteen minutes. The quinone chlorimine being also slightly volatile, and tending to decompose on long standing in acid solution, it is necessary to add a small correction to account for this. Any error introduced by the action of the dissolved air on the iodide subsequently added is included in this correction, which averages 1.5 per cent. of the total quinone chlorimine present for an aeration of from fifteen to twenty minutes.

The details of the method finally adopted are shown in the following examples of its application :

Estimation of $p$-Aminophenor, $p$-Phenetidine, otc.-An amount of an acid solution equivalent to about $0.1 \mathrm{grm}$. of the base is measured into a 250 c.c. stoppered bottle and diluted to rather more than 100 c.c. ; 5 c.c. of strong hydrochloric acid are added, followed by 10 c.c. of sodium hypochlorite solution (about $0.8 \mathrm{~N}$ ). The resulting solution should be pure yellow, and not deposit yellow flocks. Air is now blown through at a brisk rate for fifteen minutes, in which time all chlorine will have been removed, 2.5 grms. of potassium iodide are added, and the solution allowed to stand for at least five minutes, as the reduction is rather slow. The liberated iodine is then titrated with $\frac{N}{10}$ thiosulphate and starch indicator. Any residual blue tint shows that reduction has not been complete.

Each c.c. of $\frac{\mathrm{N}}{10}$ thiosulphate is equivalent to $0.00273 \mathrm{grm}$. of $p$-aminophenol, or $0.00343 \mathrm{grm}$. of $p$-phenetidine. The result is multiplied by the factor 1.015 to correct for loss during aeration.

Estimation of Phenacetin.-One grm. of phenacetin is boiled for two hours with a mixture of 25 c.c. strong hydrochloric acid (1.16) and 15 c.c. water in a small flask fitted with an air condenser. After cooling, the solution is diluted to some definite volume, and an aliquot representing $0.2 \mathrm{grm}$. phenacetin is taken for estimation exactly as above.

Each c.c. of $\frac{\mathrm{N}}{10}$ thiosulphate is equivalent to 0.00448 grm. of phenacetin. A large number of samples of commercially pure phenacetin, examined as above, gave figures ranging from $99 \cdot 2$ to $100 \cdot 2$ per cent.

Estimation of Phenaceinin in Admixture.-The following experiments were made on mixtures of phenacetin with caffeine citrate, salol, and acetanilide respectively : 
With Caffeine Citrate.-A mixture of $0.8 \mathrm{grm}$. phenacetin with $0.4 \mathrm{grm}$. caffeine citrate was treated exactly as for phenacetin. The results were slightly high, owing to formation of a small amount of some substance from the caffeine citrate which liberated iodine from hydriodic acid. The percentage of phenacetin calculated out 68.5 and $68 \cdot 7$, instead of 66.7 required by theory. No correction for the volatility of the quinone chlorimine was made in these cases, the error already mentioned more than compensating for loss by this means.

With Salol.-A mixture of equal parts of phenacetin and salol (0.5 grm. of each) was dissolved in 20 c.c. 10 per cent. sodium hydroxide, and warmed on the steambath for fifteen minutes to hydrolyse the salol; 40 c.c. of strong hydrochloric acid were then added, and the mixture boiled for two hours. The hydrolysed solution was shaken with ether to remove salicylic acid and phenol, and the chlorination and titration carried out in the usual manner. The amount of phenacetin found was $49 \cdot 3$ per cent. It was found necessary to remove the products of hydrolysis of salol before adding the hypochlorite, as the precipitates they formed with this reagent held back chlorine and caused high results to be obtained.

With Acetanilide.-Mixtures of acetanilide and phenacetin cannot be analysed without first removing the acetanilide, as the aniline produced from this substance forms an oily precipitate which apparently retains free chlorine.

Estimation of other $p$-Phenetidine or $p$-Aminophenol Derivatives.Lactophenin (lactyl-p-phenetidine) and salophen (salicylic ester of acetyl-p-aminophenol) were both estimated after hydrolysis in exactly the same manner as phenacetin, lactophenin giving 99.3 per cent. and salophen 100.4 per cent., after adding the correction previously mentioned.

Analysis of Photographic Defelopers.-In addition to the medicinal substances already mentioned, there are several $p$-aminophenol derivatives largely used in photography as developers, which may be estimated in the same manner. For instance, in a developer of the rhodinal type, the proportion of $p$-aminophenol may be quickly found by direct treatment of the acidified solution, the sulphite present being oxidised by the excess of chlorine added.

In the case of metol (methyl-p-aminophenol sulphate) it is interesting to note that, owing to the presence of the methyl group in the amino-group, no chlorination of the latter takes place, although a quinone derivative is formed. The reaction is probably

$$
\text { OH. } \mathrm{C}_{6} \mathrm{H}_{4} \cdot \mathrm{NH} \cdot \mathrm{CH}_{3}+2 \mathrm{Cl}=\mathrm{O}: \mathrm{C}_{6} \mathrm{H}_{4}: \mathrm{N}\left(\mathrm{CH}_{3}\right)+2 \mathrm{HCl} \text {. }
$$

Consequently, in the subsequent oxidation of hydriodic acid, only two atoms of iodine are liberated per molecule instead of four, as in the case of $p$-aminophenol. The reaction therefore provides a simple means of distinguishing between metol and "metol substitutes," as all the substitutes that I have examined have proved to be either $p$-aminophenol or $p$-aminocresol salts, none of them showing evidence of the presence of a methyl group substituted in the amino group, when tested by the quinone chlorimine method.

I desire to express my thanks to Messrs. Boots' Pure Drug Company, in whose laboratories the above work was carried out. 